\title{
An optimized 2.4 GHz VCO circuit design and simulation with high-Q MEMS LC-tank
}

\begin{abstract}
This paper focuses on design of high-performance MEMS LC-tank circuit for use in CMOS voltage controlled oscillators (VCO) operating at $2.4 \mathrm{GHz}$. The high-Q air suspended inductor has been designed by inductance of $2.87 \mathrm{nH}$ using MEMS technology to reduce the resistive loss and the substrate loss. A MEMS two-gap tunable capacitor has been designed. The DC voltage is $2.5 \mathrm{~V}$ which is applied to the plates and the results of $2.04 \mathrm{pF}$ could be achieved. The pull-in voltage has been optimized to achieve low phase noise, low power consumption VCO. Through this optimization, less phase noise $(-117.7 \mathrm{dBc} / \mathrm{Hz}$ at $100 \mathrm{KHz})$ and lower power consumption (11 $\mathrm{mW}$ ) have been obtained.
\end{abstract}

Keyword: MEMS LC Tank, VCO, Inductor, Varactor, Q Factor 